\title{
Strategies for Thermal Control of a Multifunctional Power Structure Solar Array
}

\author{
J. A. Foster ${ }^{1}$ and G. S. Aglietti ${ }^{2}$
}

\begin{abstract}
Multifunctional power structures (MFPS) are fully integrated subassemblies that perform both structural and power functions for spacecraft. By combining functions across subsystems into single units, mass and volume savings can be achieved. Focusing on battery-based MFPS in Earth-orbiting spacecraft, the embedded lithium ion batteries that are used have strict temperature limits, outside of which efficiency and safety is compromised. Considering the limits of the model's prediction accuracy, numerical simulation has shown that a range of Earth orbits exist where an MFPS mounted in a deployed solar array would not require the addition of further thermal control with respect to the nonmultifunctional case. The numerical simulation consisted of a lumped parameter reduction of the model to a discrete set of layers. Thermal control is required to prevent overcooling of the battery in eclipse and to extend the range of orbits where MFPS can be used. An assessment of current thermal control was performed to establish the viability of each technology, with viability defined by feasibility and the mass of the system. The use of coatings, insulation, heaters, and phase-change materials were considered. It was found that the range of viable orbits is dependent on the quantity of MFPS savings that can be sacrificed. DOI: 10.1061/(ASCE)AS.1943-5525.0000139. ( 2012 American Society of Civil Engineers.
\end{abstract}

CE Database subject headings: Spacecraft; Thermal factors; Solar power; Space structures.

Author keywords: Spacecraft thermal control; Multifunctional Structures; Lithium batteries.

\section{Introduction}

A multifunctional power structure (MFPS) is a spacecraft component that performs the functions of both power storage and structural support. The removal of the discrete components that separately perform these tasks may provide a mass benefit to the spacecraft. A discrete battery pack requires a tertiary structure to support, contain, and mount it to the spacecraft. This is a parasitic mass that adds no functionality to the spacecraft. Aside from mass, the bus volume of the spacecraft may be reduced by using an MFPS, as volume inside the spacecraft is no longer required for the batteries which can be integrated into the volume of the structural elements. A further mass savings can be achieved if the powerstorage material directly carries structural loads, allowing a reduction in the required structure. A qualitative analysis of these benefits has been performed by Roberts and Aglietti (2008).

There are two implementations of the MFPS principle. The first involves the development of a purpose-built component. This is the more efficient MFPS, as it can be optimized to the specific application. Snyder et al. (2007) of the Army Research Lab are working on developing a battery chemistry with good structural properties, but have yet to produce a component that has sufficient capability to fulfill either function. ITN Energy Systems (Neudecker et al. 2003) have developed a thin-film battery that is wrapped around individual carbon fibers and Boundless (Olson et al. 2003) have

\footnotetext{
${ }^{1}$ Astronautics Research Group, Univ. of Southampton, Southampton SO17 1BJ, UK (corresponding author). E-mail: jaf101@ soton.ac.uk

${ }^{2}$ Astronautics Research Group, Univ. of Southampton, Southampton SO17 1BJ, UK.

Note. This manuscript was submitted on June 16, 2010; approved on May 26, 2011; published online on May 28, 2011. Discussion period open until December 1, 2012; separate discussions must be submitted for individual papers. This paper is part of the Journal of Aerospace Engineering, Vol. 25, No. 3, July 1, 2012. CASCE, ISSN 0893-1321/2012/3-454-462/ $\$ 25.00$.
}

combined a bicell with aluminum to create a sandwich panel that stores power, both of which can be used to create structures.

These approaches suffer from being purpose-built for the application. In the space industry, production runs are very small and the costs of using nonstandard parts increases greatly, particularly the tooling costs. Nonstandard parts that may be handmade suffer from inconsistent quality and performance, requiring the designer to make allowances for underperformance, normally by adding redundancy — which in turn adds mass. This issue can be partially resolved through the use of commercial off-the-shelf (COTS) parts, as these parts are cheaply mass-produced to consistent quality standards.

Thus, the second implementation is a COTS MFPS, as proposed by Roberts and Aglietti (2008). It consists of polymer lithium ion (PLI) (Ilic et al. 2004) cells embedded into the honeycomb core of a sandwich panel, reducing optimization but lowering cost. PLI cells are used because they have high specific capacity and are available in prismatic shapes, making them easier to mount inside the core. The cost savings enabled by the mass savings can only be realized if COTS MFPS are shown to be viable for use on spacecraft. In pursuit of this, Roberts has addressed the issues of manufacture (Roberts and Aglietti 2007), launch vibration (Roberts and Aglietti 2006), and the effect the batteries have on the structural performance of the panel (Foster et al. 2008). Wang et al. (2006) have shown that PLI cells can survive the radiation and vacuum encountered in space. Choquette and Lessard-Deziel (2002) have assessed the lithium polymer batteries for the effects of proton and electron radiation and concluded that over the course of a 5-year mission, the batteries would suffer no significant loss of performance.

One issue that has not specifically been addressed is that of temperature management of the batteries for a space application. Outside of its temperature envelope, the battery suffers loss of efficiency and permanent damage if the limits are greatly exceeded (Chen and Evans 2005). When the COTS MFPS is used as part of the main body of the spacecraft and is internal, the spacecraft's 
thermal-control system can maintain battery temperature. However, if the COTS MFPS forms part of a structure that is external to the spacecraft, i.e., as part of a solar array, it may require thermal control to maintain battery temperature.

Various researchers (Megahed and El-Dib 2007; Queheillalt et al. 2001; Queheillalt et al. 2008) have presented multifunctional thermal structures of which the purpose has been to increase the thermal-control functionality of a structure, such as embedding heatpipes into a panel. The NASA New Millenium Project Deep Space 1 spacecraft contained a multifunctional structure, and part of the experiment was to test the thermal control. However, this was an electronics-based component, not power-storage, and the thermal-control issue was dissipating the heat emitted by small components (Rawal 1999). The thermal control of PLI batteries has been discussed by Khateeb et al. (2005) and Hisano et al. (1995), though these are terrestrial applications considering an electric scooter and a laptop computer. Previous work by the authors (Foster and Aglietti 2010) has shown that, while there are orbits in which no thermal control is needed, there are many orbits in which it is needed. The purpose of this paper is to close the research gap between thermal-control and MFPS by assessing the viability of possible thermal-control solutions and to extend the range of orbits in which MFPS can be used.

\section{Multifunctional Solar Array}

As developed by Foster and Aglietti (2010), the application considered is that of an MFPS embedded into the structure of a deployed-wing solar array. Such a design places the batteries in close proximity of the solar cells; with the use of thin-film electronics and multichip modules, this could provide an additional mass reduction in the electrical power system, though this is not the focus of this paper. However, placing batteries in a deployed-wing solar array leaves them very exposed to the environment. To assess the thermal technical issue, an application was developed for numerical modeling that is typical of current satellite technology. The spacecraft to which the array is attached is not modeled to avoid overconstraining the model and to represent the worst-case scenario in which thermal control of the MFPS cannot rely on support from the spacecraft bus.

The case in which the MFPS solar array is body-mounted is not considered in this paper for this very reason. Whether mounted to the outside of the spacecraft via flexures or forming part of the main body of the spacecraft, when the panel is body-mounted, it has access to the thermal resources of the spacecraft. The most important of these are the thermal inertia of the spacecraft which slows the response to changing environments, the number of available surfaces for the absorption and rejection of heat, and the option to use thermal control that can be outside of the MFPS panel. Lacking this resource, the wing mounting is the worst-case scenario.

The application is modeled as a single panel of the wing array, which is orientated such that the solar cells are perpendicular to the Sun's input at all times, as is standard to maximize power from the cells. In general terms, the panel is a sandwich panel that is composed of a facesheet and a core, with a battery embedded in the core, and solar cells on one face, as proposed by Roberts and Aglietti (2008), see Fig. 1.

\section{Environment}

The environment in Earth orbit is dominated by heat from the Sun and from the Earth. Two variations on the orbit are considered, a hot case representing all of the inputs at their maximum, and a cold case representing all of the inputs at their minimum. These environments

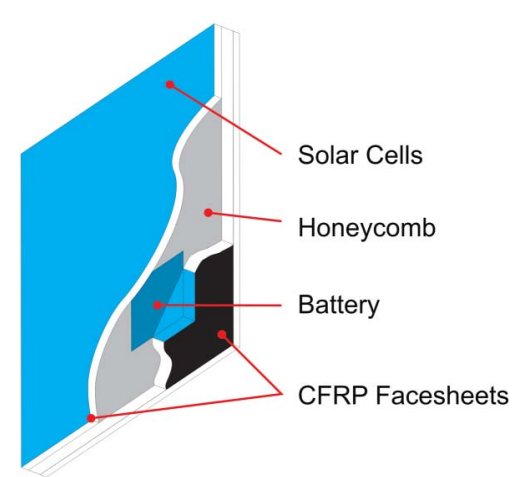

Fig. 1. Schematic of proposed MFPS application

are the extremes that the MFPS panel can be expected to encounter in Earth orbit. The Sun is modeled as an input of $1414-$ $1322 \mathrm{~W} / \mathrm{m}^{2}$ (Gilmore 2002) on the solar cells. The Sun's input is considered to be parallel to the Earth-Sun vector for the calculation of angles of incidence. The shadow cast by the Earth is modeled as a cylinder with a radius equal to that of the Earth.

The Albedo model used (Gilmore 2002) was developed by NASA (Anderson et al. 2001; Justus et al. 2001). Averaging data from a number of experiments, the model takes the form of look-up tables, in which for a time constant of hundreds of seconds and for surfaces susceptible to both infrared and Albedo, the $3.3 \sigma$ values for the reflection of sunlight by the Earth's surface is 0.14 at minimum and 0.28 at maximum. These values are corrected for the effects of the angle of incidence between the Sun's rays and the surface of the earth and corrected for the effect of non-Lambertian reflection near the terminator using the given factors (these are dependent on the solar-zenith angle at the surface above which the spacecraft ). From the same NASA model using the same inputs, the minimum infrared heat from the Earth is $232 \mathrm{~W} / \mathrm{m}^{2}$ and the maximum is $275 \mathrm{~W} / \mathrm{m}^{2}$. These values have been normalized for an altitude of $30 \mathrm{~km}$ above the Earth's surface and are propagated from there using an inverse-square rule.

The panel radiates in the infrared spectrum to cold space, which has a temperature of $4 \mathrm{~K}$. The power absorbed by the solar cells is sent to the spacecraft with none being remitted inside the MFPS panel. The batteries are considered to be trickle-charged and thus to produce only a negligible amount of heat.

\section{Lumped-Parameter Modeling}

A numerical model of the MFPS panel was created using ThermXL, which is a spreadsheet-based program that uses lumped-parameter modeling. The model is formed of nodes with heat-flow paths defined between them. The nodes are used in four different ways: boundary nodes are used to represent a surface, having the optical properties of that surface and interacting with the thermal environment. A fixed node is used to represent cold space. Mass nodes represent the mass and heat capacity of an amount of material. Interface nodes are used to allow the changing of conduction properties between materials. Conduction heat flow is defined by Eq. (1), where $Q_{k}=$ heat flow; $k=$ thermal conductivity; $A_{k}=$ cross-sectional area; $x=$ path length; and $T_{i}, T_{j}=$ node temperatures. Radiation heat flow from a surface to cold space is defined by Eq. (2), where $Q_{r}=$ heat flow; $\varepsilon_{i}=$ emittance of the surface; $\varepsilon_{j}=$ emittance of cold space assumed to be 1 ; $A=$ area of the surface; and $F_{\mathrm{ij}}=$ view factor between the surface and cold space, also assumed to be 1 . 


$$
\begin{gathered}
Q_{k}=\frac{k A_{k}}{x}\left(T_{i}-T_{j}\right) \\
Q_{r}=\varepsilon_{i} \varepsilon_{j} A_{r} F_{i j}\left(T_{i}-T_{j}\right)
\end{gathered}
$$

The panel is modeled as a set of layers, in which each layer is modeled by a conduction path with three nodes: a mass node to represent the mass and heat capacity of the layer and an interface node at each end of the path. Interface nodes are shared between layers. Thus, only heat flow through the panel is considered, not across it. The Biot number for each layer is close to or less than 0.1 , indicating that thermal gradients across each layer are small and that important information on the flow of heat is not lost. Considering only heat flow through each layer removes detail of the temperature distribution across each layer. However, as there is no heat flow across the panel edges, the temperature distribution across each layer is uniform.

The model does not consider how the batteries are distributed throughout the core, only that they are of a consistent depth, as only the through properties of each layer are considered. As such, two paths exist between the carbon-fiber-reinforced polymer (CFRP) facesheets, one through honeycomb and one through the batteries. These paths are shown in Fig. 2; the path through the batteries goes through nodes $3,4,5,6,7,8$, and 10 , with a ratio of cross-sectional area between this and the path through the honeycomb core (nodes 3,9 , and 10) of 0.46 . The geometry of the honeycomb affects the conductive flow of heat. For heat flow through the honeycomb layer, the effect can be modeled by considering the actual crosssectional area of honeycomb, which is $4.2 \%$ of the area covered by the core. Radiation exchange inside the honeycomb is not modeled, as it is $2-4$ orders of magnitude smaller than the heat flow of conduction.

The solar cells are not modeled directly as they are too thin for the modeling software. The resulting conduction paths cause numerical instability as a result of the large differences in orders of magnitude. The solar cells are thus treated as a smeared mass over the surface of the Sun-side facesheet and their heat capacity and optical properties are added (applied) to this facesheet. Because the solar cells have a high thermal conductivity, they would not

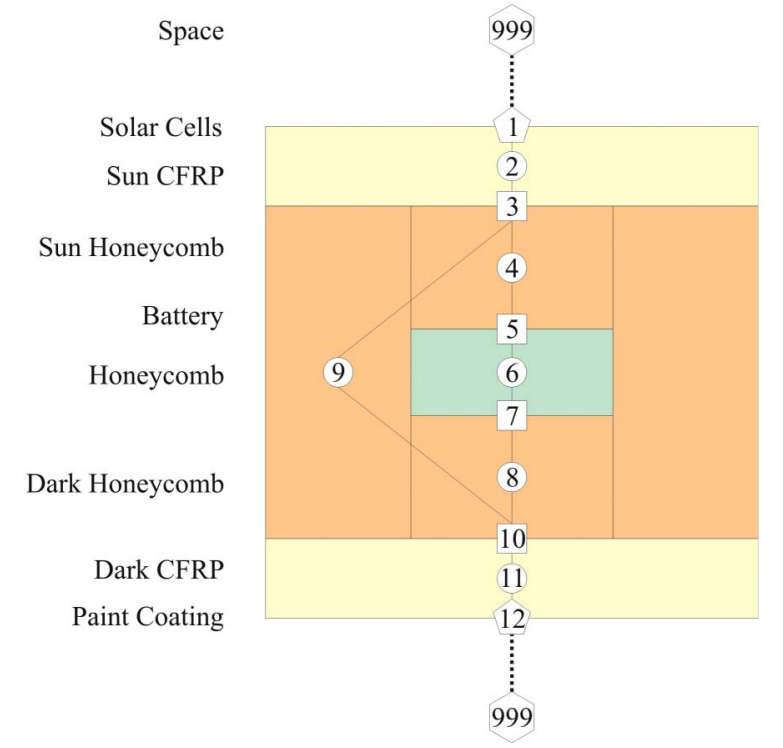

Fig. 2. Finite-difference model of wing mounting. Hexagons represent fixed nodes, pentagons represent boundary nodes, squares represent interface nodes, and circles represent the mass nodes have insulated the panel, and thus the removal of this layer will produce only a minimal increase in heat flow to the rest of the panel. Space is modeled as a node with a radiation connection to the boundary nodes. Kirchoff's law (Reif 1965) is used for radiation in the infrared range, and it is assumed that the heat radiation given off by both the Earth and surfaces is in the infrared range. As such, when considering infrared radiation, the emittance is equivalent to the absorptance. No orbit perturbation is modeled because its effect is indirect (inputs are a function of position) and it would add unnecessary complexity. Celestial bodies (i.e., the Earth and the Sun) are considered fixed to reduce complexity.

The effects of the assumptions used to build the numerical model are to simplify the simulation to a small number of nodes. As described above, each assumption has been shown to have, at worst, a nearly negligible effect on the flow of heat through the panel and no effect on the heat capacity of the panel or the boundary conditions. As such, high prediction accuracy is expected of the model, though all results from the model should be considered in terms of the reduced resolution to which the assumptions lead.

The wing model is composed of 13 nodes, 11 conduction paths, and 2 radiation paths. A schematic diagram of the model is shown in Fig. 2. The radiation paths link the boundary nodes 1 (solar-cell surface) and 12 (paint-coated surface) to fixed node 999 (cold space). The Sun's radiation is incident on node 1. Albedo and infrared radiation from the Earth is incident of the surface node that is facing the Earth-node 12 when the panel is between the Earth and the Sun, and node 1 when the Earth is between the panel and the Sun. To summarize the specific thermal boundary conditions, the model emits radiation from nodes 1 and 12 to node 999, which has a fixed temperature.

\section{Numerical Definition of Model}

Table 1 shows the details of the panel and the variables assigned to the properties. The panel is assumed to have been manufactured to its nominal properties. As such, no gaps exist, and contact conduction is not considered to be a factor in the flow of heat. Because the panel is manufactured under pressure with an adhesive, and it is an important structural requirement that the core is bound to the facesheet, this is deemed a reasonable assumption. A 100\% packing factor for the solar cells is used to simplify the model; the entire Sun-facing surface is a solar cell. This also represents the worstcase design scenario in which there are fewer design options as the surface properties of gaps between solar cells cannot now be optimized.

The core geometry is hexagonal and has the following properties: $3.175 \mathrm{~mm}, 0.051 \mathrm{~mm}, .113 \mathrm{~kg} / \mathrm{m}^{3}$, and AL5052. The Kapton tape added to the edges of the panel reduces the heat transfer across this very small area to negligible amounts. The adhesives used to bond the materials together are considered to be a submillimeter in thickness and are therefore considered to have no effect. The dark surface of the panel is given a paint coating or surfacing, the optical properties of which can be altered. The thermal properties of the materials used are given in Table 2.

The battery was selected for its superior properties (Ilic 2004). The temperature envelope is $273-318 \mathrm{~K}$, selected for a conservative model. This is potentially a wide temperature range, as Gilmore (2002) notes that it is preferential for $\mathrm{NiCd}$ and $\mathrm{NiMH}$ batteries to be kept between $273 \mathrm{~K}$ and $298 \mathrm{~K}$ for better efficiency. Tighter constraints will require greater thermal-control authority and may reduce viability.

To meet the power-storage requirement, a number of battery cells are required. It is safe to assume that the solar-cell area of a spacecraft is the minimum required, as extra solar cells would not improve the performance of the spacecraft. Thus, the solar-cell 
Table 1. Properties of Example MFPS Application

\begin{tabular}{|c|c|c|c|}
\hline Property & Variable & Value & Unit \\
\hline Facesheet material & & CFRP & \\
\hline Fibers & & M55J & \\
\hline Resin & & Epoxy & \\
\hline Volume fraction & $V_{f}$ & 0.6 & \\
\hline Biweave ply thickness & $X_{\text {ply }}$ & 0.3 & $\mathrm{~mm}$ \\
\hline Facesheet thickness & $X_{f}$ & 1.8 & $\mathrm{~mm}$ \\
\hline Core type & & Honeycomb & \\
\hline Core material & & Aluminium & \\
\hline Core thickness & $X_{c}$ & 12 & $\mathrm{~mm}$ \\
\hline Solar cells & & Triple-junction GaAs & \\
\hline Solar-cell efficiency & $\eta_{s c}$ & $28 \%$ at $301 \mathrm{~K}$ & \\
\hline Solar-cell temperature coefficient & $\eta_{T}$ & 0.07 & $\mathrm{~K}^{-1}$ \\
\hline Solar-cell absorptance & $\alpha_{s c}$ & 0.91 & \\
\hline Solar-cell emittance & $\varepsilon_{s c}$ & 0.8 & \\
\hline Battery cell & & Varta PLF263441 D & \\
\hline Cell power density & $P_{\rho}$ & 166 & $\mathrm{Wh} / \mathrm{kg}$ \\
\hline Cell mass & $M_{c}$ & 6 & $\mathrm{~g}$ \\
\hline Number of cells & $N_{b}$ & 900 & \\
\hline Cell total mass & $M_{c t}$ & 5.4 & $\mathrm{~kg}$ \\
\hline Total energy capacity & E & 1 & KWh \\
\hline Kapton tape absorptance & $\alpha_{\mathrm{kt}}$ & 0.12 & \\
\hline Kapton tape emittance & $\varepsilon_{\mathrm{kt}}$ & 0.03 & \\
\hline Panel area & $A_{w}$ & 1 & $\mathrm{~m}^{2}$ \\
\hline Coating absorptance & $\alpha_{\mathrm{wp}}$ & 0.09 & \\
\hline Coating emittance & $\varepsilon_{\mathrm{wp}}$ & 0.92 & \\
\hline Panel mass & $M_{w}$ & 8.4 & $\mathrm{~kg}$ \\
\hline Panel heat capacity & $H_{w}$ & 7.86 & $\mathrm{~kJ} / \mathrm{K}$ \\
\hline
\end{tabular}

Table 2. Thermal Properties of Materials Used

\begin{tabular}{lcccc}
\hline & $\begin{array}{c}\text { Conductivity } \\
\text { Material }\end{array}$ & $\begin{array}{c}\text { Conductivity } \cdot \mathrm{m}^{-1} \cdot \\
\mathrm{K}-1\end{array}$ & $\begin{array}{c}\text { Conductivity } \cdot \mathrm{m}^{-1} \cdot \\
\mathrm{K}^{-1}\end{array}$ & $\begin{array}{c}\text { Specific heat } \\
\mathrm{K}^{-1}\end{array}$ \\
\hline Solar cells & 58.6 & 58.6 & 58.6 & $\begin{array}{c}\text { capacity } \\
\mathrm{J} \cdot \mathrm{kg}^{-1} \cdot \mathrm{K}^{-1}\end{array}$ \\
CFRP & 0.49 & 93.5 & 93.5 & 1048 \\
Battery & 0.72 & 62.7 & 62.7 & 803 \\
Aluminium & 138 & 138 & 138 & 880 \\
alloy & & & & \\
\hline
\end{tabular}

Note: $X$ is defined as the thickness of the panel, with $Y$ and $Z$ orthogonal to $X$.

area can be used to calculate the amount of power that the spacecraft requires. To avoid having to recalculate the number of batteries for each altitude, geosynchronous eclipse was considered, as it is the longest. The following parameters were assumed to be realistic: power use in eclipse, $80 \%$; charging efficiency, 80\%; eclipse time, $1.12 \mathrm{~h}$; eclipse fraction, 0.047; depth of discharge, $40 \%$;

The total solar-array area for the spacecraft is unknown, as the system around the isolated panel has not been described. However, the minimum solar-cell area would be the panel by itself. This gives a solar-power requirement of $271 \mathrm{~W}$ and an eclipse power of $217 \mathrm{~W}$. This requires a capacity of $821 \mathrm{Whr}$ and 740 batteries. For a level of redundancy (to address degradation with age and faults), 900 batteries are used to give $1 \mathrm{kWhr}$ of capacity. Roberts and Aglietti (2008) have defined the savings from using MFPS, however the

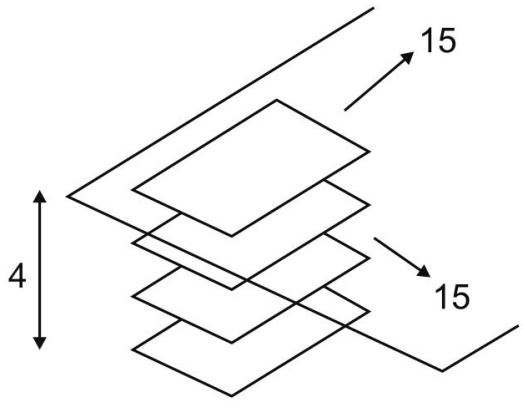

Fig. 3. Diagram of the arrangement of the batteries in the panel

spacecraft is undefined and thus calculation of the specific energy requirement is difficult. However, assuming the spacecraft is $250 \mathrm{~kg}, 500 \mathrm{~kg}$, or $1000 \mathrm{~kg}$, gives specific energy requirements of 4,2 , and 1 . This, in turn, gives a savings of $1.18 \%, 0.59 \%$, and $0.3 \%$, corresponding to a mass savings of $2.95 \mathrm{~kg}$ in all three cases. The batteries are arranged $15 \times 15 \times 4$ in the panel, as shown in Fig. 3, and are located at the center of the honeycomb core. This would be difficult to manufacture, as a separate splicing of the core would be required, in addition to a more complex assembly, to ensure the batteries and core subsections were correctly mounted inside the panel to preserve structural properties.

\section{Baseline Results}

For both environments, a transient modeling of the wing mounting at $200 \mathrm{~km}$ and geosynchronous orbit performed. A circular low earth orbit (LEO) at $200 \mathrm{~km}$ will experience a short eclipse and pass through the overheating region at its greatest, therefore testing if this short, sharp heating/cooling regime will cause the battery to leave its temperature envelope. A geosynchronous earth orbit (GEO) was modeled to test if the panel will overcool in eclipse and if the panel will reach its equilibrium temperature when sunlit away from Earth. In reality, these orbits would have properties that would give a Sunsynchronous LEO and a geosynchronous orbit about the Earth'sequator, these being representative of typical orbits at these altitudes. In this work, the orbits are not defined beyond being circular and the longitude of the ascending node causing the orbit to pass over the subsolar point (a right ascension of the ascending node of $0^{\circ}$ ), the requirement being for the longest possible eclipse. In the following figures, $\mathrm{HC}$ stands for the honeycomb core.

The results in Figs. 4-7 agree well with those presented in Li et al. (2006) and Foster and Aglietti (2010). The LEO results

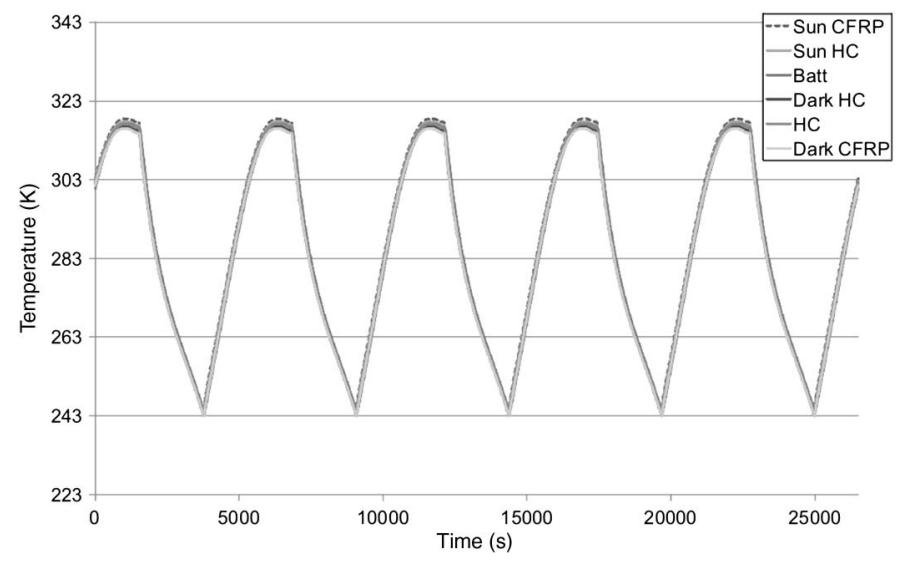

Fig. 4. Lumped-parameter transient results of the wing panel in a hot LEO case 


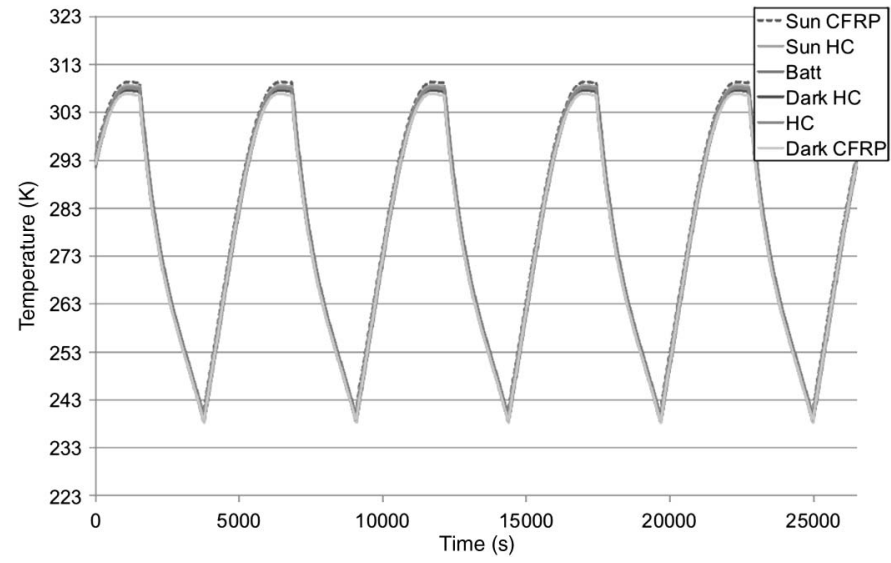

Fig. 5. Lumped-parameter transient results of the wing panel in a cold LEO case

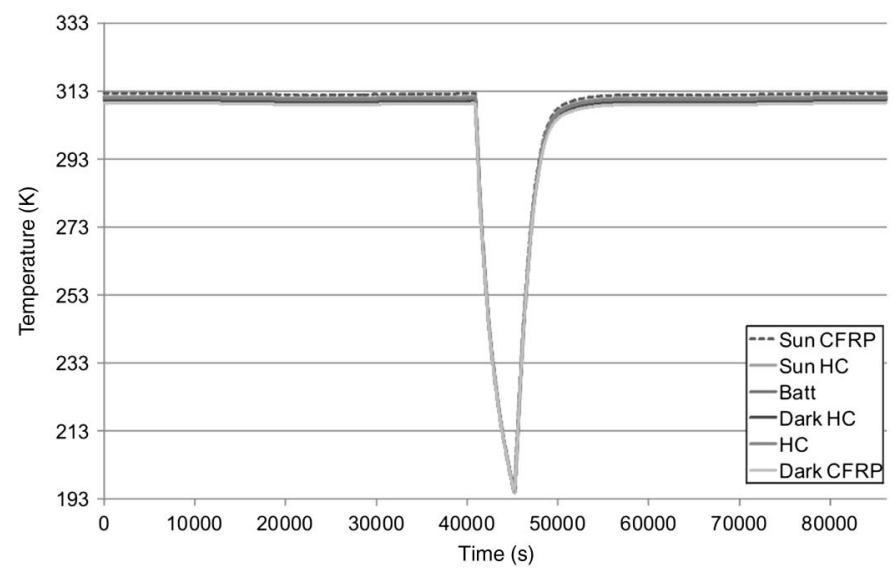

Fig. 6. Lumped-parameter transient results of the wing panel in a hot geosynchronous case

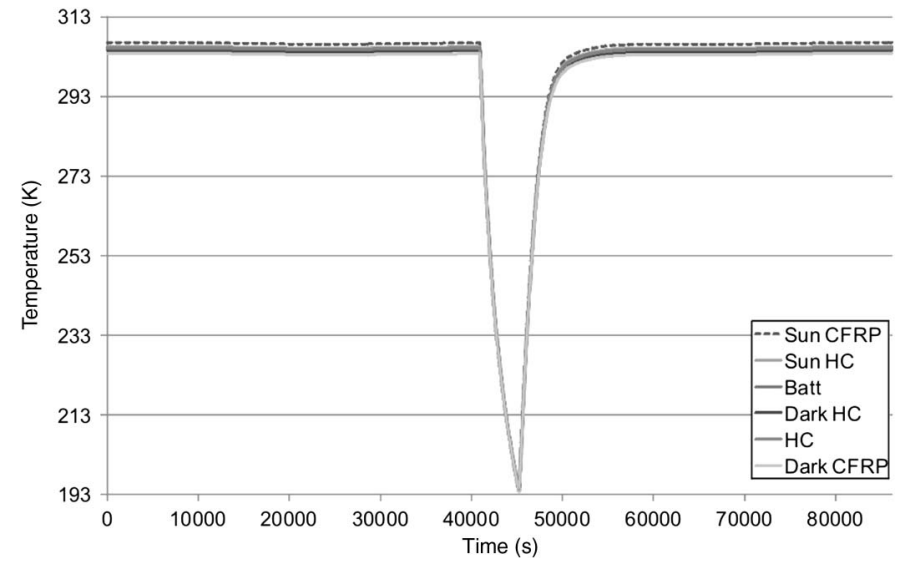

Fig. 7. Lumped-parameter transient results of the wing panel in a cold geosynchronous case

show the rapid change caused by the short orbit period. The geosynchronous temperatures reach equilibrium in sunlight and experience a plunge in temperature during eclipse. All of the results show that the temperature difference through the panel is at most $3 \mathrm{~K}$. Overcooling occurs in all orbits and is worst at geosynchronous altitude where the eclipse is longest and there is no input from the Earth. Table 3 shows the time between eclipse entry and overcooling occurring. Orbits that do not require thermal control to prevent overcooling can be defined as those where the eclipse is shorter than the time to overcool.

Overheating does not occur in any of the considered orbits, though in the hot LEO case, the battery comes very close to the upper limit of $318 \mathrm{~K}$. This is a clear indication that there exists a time to overheating for orbits that spend longer above the subsolar point where the heat input is at a maximum. Time above the subsolar point and in eclipse is reduced with an increasing angle between the solar and orbit planes (the $\beta$ angle). For MFPS to be used in orbits with low $\beta$ angles, thermal control is required.

\section{Thermal Control Viability}

The viability of a thermal-control system (TCS) is defined in this paper by two factors. In addition to the obvious requirement of it being feasible that the thermal-control method maintains battery temperature, the mass of the TCS must be less than the savings gained from using an MFPS for the advantage of using MFPS to be realized.

\section{Coatings}

The effects of varying the optical properties of the coated surface of the panel have already been partially considered by Foster and Aglietti (2010); the performance as a TCS is specifically considered here. The emittance of the surface defines a large proportion of the heat loss from the panel. Table 4 shows the minimum emittance required to prevent overheating and the maximum emittance that prevents overcooling. These requirements are in conflict and there exists no range of emittances that prevent both overcooling and overheating. Varying the emittance gives an increase on the time to overcool over the baseline results by lowering the emittance to the minimum to prevent overheating. Table 5 shows the new times to overcool, where the effect is greatest in the cold orbits as the minimum emittance to avoid overheating can be lower.

Table 3. Times to Overcool

\begin{tabular}{lcc}
\hline Orbit & Hot environment & Cold environment \\
\hline $200 \mathrm{~km} \mathrm{LEO}$ & $890 \mathrm{~s}$ & $722 \mathrm{~s}$ \\
GEO & $630 \mathrm{~s}$ & $560 \mathrm{~s}$ \\
\hline
\end{tabular}

Table 4. Minimum and Maximum Values of Emittance to Prevent Overheating and Overcooling

\begin{tabular}{lcc}
\hline Case & Minimum for overheating & Maximum for overcooling \\
\hline LEO hot & 0.90 & 0.32 \\
LEO cold & 0.66 & 0.25 \\
GEO hot & 0.77 & No value \\
GEO cold & 0.66 & No value \\
\hline
\end{tabular}

Table 5. Times to Overcool with Minimum Emittance to Avoid Overheating

\begin{tabular}{lcc}
\hline Orbit & Hot environment & Cold environment \\
\hline $200-\mathrm{km} \mathrm{LEO}$ & $920 \mathrm{~s}$ & $1150 \mathrm{~s}$ \\
Geosynchronous & $840 \mathrm{~s}$ & $950 \mathrm{~s}$ \\
\hline
\end{tabular}


As the panel will always have a coating on the shadowed side of the panel, there is no added mass.

\section{Variable Emittance}

Variable-emittance solutions, such as louvers, enable the switching between potential emittances for the coated surface. Louver are only considered in LEO, as it has been shown that no emittance exists that can prevent overcooling in GEO. Fig. 8 shows a louver that switches between the theoretical limits of emittance ( 0 and 1) for a range of switch temperatures in a hot LEO. Even with the best emittances, it can be seen that a louver is not a feasible solution. When the best emittance to optimize the heat loss is used, the entry temperatures into sunlight and eclipse are too close to the limits and thus the battery temperature becomes unsafe.

Fig. 9 shows a louver that switches between the best physically possible emittances (0.04 and 0.94) in a cold LEO. From the range of switch temperatures considered, such a louver is feasible if the switch temperature is above $303 \mathrm{~K}$; the cooler environment allows it to exit eclipse at $273 \mathrm{~K}$ and avoid overheating. Viability is dependent upon the louver mechanism. A traditional mechanical louver is likely to be too heavy, though louvers that use microelectro-mechanical systems may provide a low-mass solution.

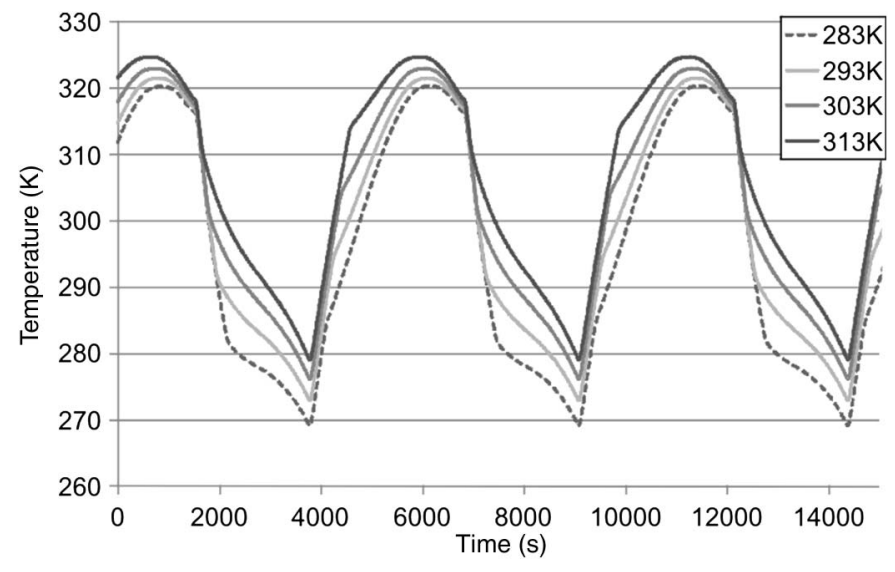

Fig. 8. The effect of switching temperature on louver performance in a hot LEO

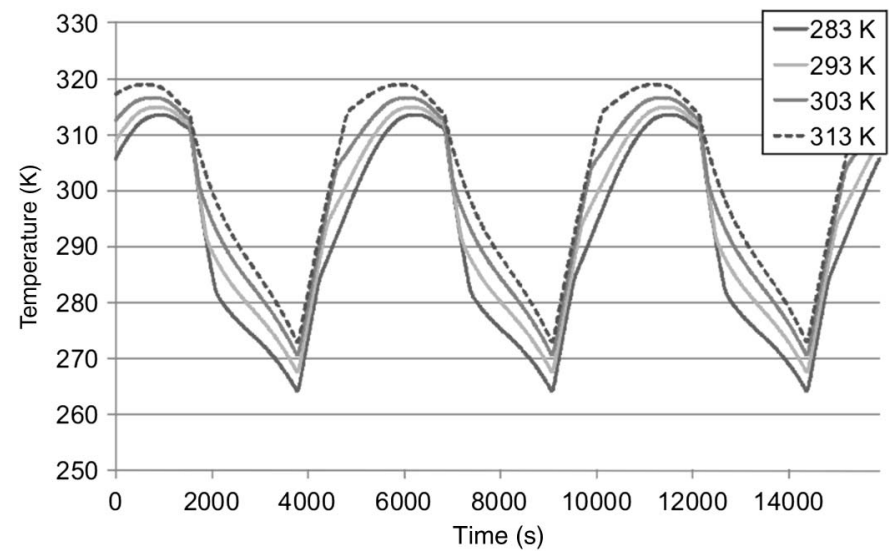

Fig. 9. The effect of switching temperature on louver performance in a cold LEO

\section{Insulation}

The battery can be insulated against the changing environment by replacing the honeycomb core with a more insulating material. A carbon aerogel (Wienar et al. 2006) is the most insulating material currently available, though a honeycomb of Nomex would also have a similar insulating effect. Using an aerogel as the core of the sandwich panel will greatly slow down the flow of heat from the battery during eclipse, increasing the time to overcool. The chosen carbon aerogel has the following thermal properties: density, $-312 \mathrm{~kg} / \mathrm{m}^{3}$; specific heat capacity, $-500 \mathrm{~J} / \mathrm{kgK}$; and thermal conductivity, $-0.005 \mathrm{~W} / \mathrm{mK}$. Directly replacing the honeycomb with aerogel adds $1.56 \mathrm{~kg}$ to the panel. Table 6 shows the new times to overcool. While providing an increase, the use of aerogel is possibly limited by its weak structural properties which may make it an unsuitable core material.

\section{Heaters}

Heaters are an active TCS that directly add warmth to the batteries during eclipse. The heater is modeled as a patch of negligible mass that is applied to the mass node of the battery layer. It activates when the temperature of the batteries drops below $278 \mathrm{~K}$, a safety margin of $5 \mathrm{~K}$ above the lower limit. Table 7 shows the heater power required and the extra battery capacity that would be needed to supply the heaters to prevent overcooling for the longest eclipses. These results are for heaters that are $100 \%$ efficient, and thus the true values can be expected to be higher. Though the use of heaters has been shown to prevent overcooling, in the hot LEO case, the warmer eclipse exit temperature means that the batteries are now at risk of overheating.

\section{Phase-Change Materials}

Exploiting the heat of fusion of a material as it changes phase between solid and liquid boosts the heat capacity over the phase-transition range. In the context of the MFPS application, the batteries could be surrounded by a phase-change material (PCM), the freezing of which slows the cooling of the batteries in eclipse such that overcooling does not occur. To assess initial feasibility, the PCM is modeled as a heat capacity increase of the battery layer mass node. The change in heat capacity is modeled as a Gaussian distribution - this is a simple match for how the heat capacity changes during the transition between states. The cutoff is at $3 \sigma$, which gives a $5-\mathrm{K}$ range using Eq. (3), where $H=$ heat capacity and $T=$ temperature. Table 8 shows the masses of 10 selected PCMs. The amount of PCM required is defined as the amount such that the PCM completes freezing at eclipse exit.

Table 6. Times to Overcool Caused by Added Insulation

\begin{tabular}{lcc}
\hline Orbit & Hot environment & Cold environment \\
\hline $200-\mathrm{km}$ LEO & $1520 \mathrm{~s}$ & $1200 \mathrm{~s}$ \\
Geosynchronous & $1120 \mathrm{~s}$ & $990 \mathrm{~s}$ \\
\hline
\end{tabular}

Table 7. Requirements for the Use of Heaters to Prevent Overcooling

\begin{tabular}{lccc}
\hline Case & $\begin{array}{c}\text { Heater power } \\
\text { W }\end{array}$ & $\begin{array}{c}\text { Battery capacity } \\
\text { Wh }\end{array}$ & $\begin{array}{c}\text { Battery mass } \\
\mathrm{kg}\end{array}$ \\
\hline LEO hot & 321 & 134 & 0.81 \\
LEO cold & 343 & 160 & 0.96 \\
GEO hot & 475 & 513 & 3.09 \\
GEO cold & 476 & 500 & 3.01 \\
\hline
\end{tabular}


Table 8. Mass of Phase Change Material Required for Worst-Case Orbits

\begin{tabular}{|c|c|c|c|c|c|c|c|}
\hline & & $\begin{array}{c}\text { Transition } \\
\text { temperature }\end{array}$ & $\begin{array}{l}\text { Heat } \\
\text { of } \\
\text { fusion }\end{array}$ & $\begin{array}{l}\text { LEO } \\
\text { cold }\end{array}$ & $\begin{array}{c}\text { LEO } \\
\text { hot }\end{array}$ & $\begin{array}{l}\text { GEO } \\
\text { cold }\end{array}$ & $\begin{array}{l}\text { Geo } \\
\text { hot }\end{array}$ \\
\hline Source & PCM & $\mathrm{K}$ & $\mathrm{kJ} / \mathrm{kg}$ & \multicolumn{4}{|c|}{$\mathrm{kg}$} \\
\hline Gilmore (2002) & Water & 273 & 333 & 3.4 & 2.6 & 10.6 & 10.4 \\
\hline Gilmore (2002) & Hydrazine & 274.4 & 393 & 3.0 & 2.4 & 9.3 & 9.0 \\
\hline Hawes et al. (1993) & Rubitherm GmbH RT5 & 279 & 156 & 10.5 & 7.4 & 25.5 & 24.9 \\
\hline Hawes et al. (1993) & $\mathrm{LiClO}_{3} \cdot 3 \mathrm{H}_{2} \mathrm{O}$ & 281.1 & 253 & & 5.1 & 16.3 & 16.0 \\
\hline USGC (1999) & Formic acid & 281.4 & 247 & & 5.3 & 16.8 & 16.5 \\
\hline Gilmore (2002) & Glycerol & 291 & 199 & & & 24.9 & 24.4 \\
\hline $\begin{array}{l}\text { Automation Creations } \\
\text { (2007) }\end{array}$ & Vanadium fluoride VF5 & 292.5 & 714 & & & 36.1 & 35.3 \\
\hline Gilmore (2002) & Polyethylene glycol & 298 & 146 & & & 38.3 & 37.5 \\
\hline Gilmore (2002) & Phosphomium chloride & 301 & 752 & & & & 7.7 \\
\hline Sharma et al. (2009) & Trimethylolethan $e+$ urea $(62.5 \%+37.5 \%)$ & 302.8 & 218 & & & & 27.2 \\
\hline
\end{tabular}

The use of PCMs is limited by the need to melt them after each eclipse. At higher transition temperatures, more PCM is required as the PCM melting begins earlier as freezing begins at higher temperatures, so the time to eclipse exit is longer. In LEO the sunlit time is short, limiting the amount of PCM that can be melted. In GEO, the transition temperature must be below the equilibrium temperature in sunlight.

$$
H=\exp \left(-1.2 \mathrm{~T}^{2}\right)
$$

Table 8 shows that the use of PCMs is feasible, but that the masses required are greater than the MFPS savings. While PCMs are not a viable solution to the longest eclipses, Fig. 10 shows the delay in the cooling time that each PCM gives per unit mass of PCM that is added to the panel. For $1 \mathrm{~kg}$ (a third of the MFPS savings), vanadium flouride (VF5) gives a delay of $1000 \mathrm{~s}$ and hydrazine gives a delay of 700 s. Fig. 10 thus shows the length of eclipses, and therefore the orbits, that can be made safe against overheating for a stated loss of MFPS savings. These results should be tempered by considering that PCMs require containment for the liquid phase. Containment is required because the honeycomb core typically has perforations to allow air to escape. The liquid phase of the PCM would thus be sucked into space. A sealed honeycomb would have to cope with the structural loads caused by any trapped air and the density change of the PCM, adding mass. A box structure would require removal of honeycomb and have to structurally replace the removed honeycomb, which will add mass. Polymer mixtures or microencapsulations are always solid, but suffer

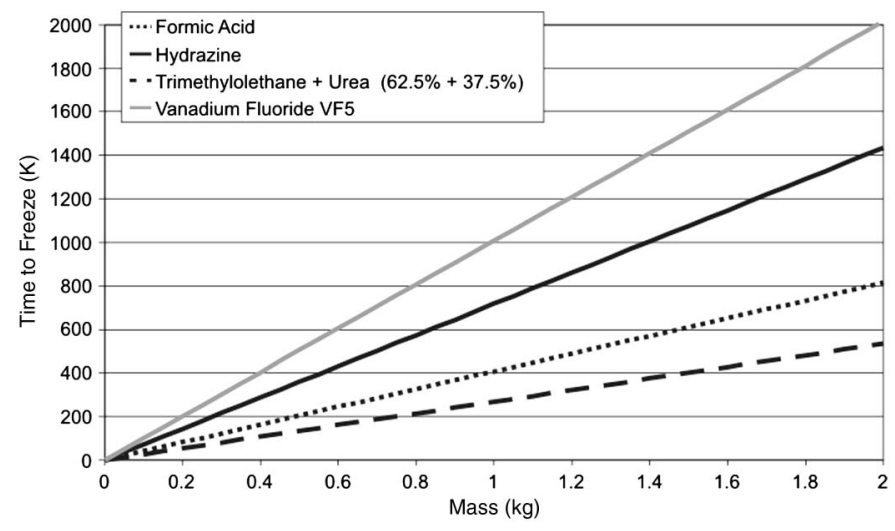

Fig. 10. The time required to freeze a selection of PCMs in geosynchronous eclipse reduced effectiveness as not all of the mass is active. In all cases, the recommendation of PCM use must be tempered by the complexities of its implementation.

\section{Discussion}

All of the considered TCS have the capability to extend the range of orbits; unfortunately, no technology is able to achieve a viable solution in all orbits. Table 9 compares the performance of the considered TCS. The table shows how efficient each system is, in terms of the increase in the time to overcool per percentage of MFPS savings used by the TCS. As coatings add negligible mass to the panel, their performance is infinite and they are considered the best-performing TCS. Table 10 shows the maximum eclipse time for which the TCS is effective. The maximum eclipse time for the insulation is the time to overcool if the entire core is replaced by an aerogel. For heaters and PCM in geosynchronous orbit, the maximum eclipse is defined as the time to overcool if all of the MFPS savings are used. For LEO PCMs, the upper limit is defined by the amount of PCM that can be melted. Details of the mass of a louver system are not available, hence their absence.

In terms of which orbits in which it is viable to use MFPS, without a particular thermal control there exists a maximum length of eclipse, which is dependent upon altitude and environment and is

Table 9. Efficiency of Each Thermal Control System in Delay Increase per $\%$ of MFPS Savings Used

\begin{tabular}{lcccc}
\hline TCS & LEO cold & LEO hot & GEO cold & GEO hot \\
\hline Coating & $\infty$ & $\infty$ & $\infty$ & $\infty$ \\
Insulation & 9 & 12 & 8 & 9 \\
Heaters & 46 & 49 & 36 & 34 \\
PCM & 29 & 21 & 29.5 & 29.5 \\
\hline
\end{tabular}

Note: Values are in s per $\%$.

Table 10. Maximum Eclipse Time in Seconds That Each TCS Can Make Safe

\begin{tabular}{lcccc}
\hline TCS & LEO cold & LEO hot & GEO cold & GEO hot \\
\hline Coating & 1150 & 920 & 950 & 840 \\
Insulation & 1200 & 1520 & 990 & 1120 \\
Heaters & 5350 & 5790 & 4125 & 4000 \\
PCM & 1630 & 2180 & 3510 & 3580 \\
\hline
\end{tabular}


defined by the time it takes the battery to become too cold. At low altitudes, orbits that have eclipses shorter than $890 \mathrm{~s}$ (hot)/720 s (cold) will not overcool and will have a $\beta$ angle such that they will not spend enough time above the subsolar point to overheat. At geosynchronous altitudes, orbits that have eclipses shorter than $630 \mathrm{~s}$ (hot)/560 s (cold) will not experience overcooling. Such orbits do not require a thermal-control system. For low Earth orbits that have eclipses shorter than $910 \mathrm{~s}$ (hot)/1150 s (cold) and geosynchronous eclipses shorter than $840 \mathrm{~s}$ (hot)/950 s (cold), applying a coating with the minimum emittance to prevent overheating is the best solution. Because the panel will always have a coating on its dark side, there will be near zero added mass.

For orbits with longer eclipses, the addition of a PCM is the most mass-efficient solution to preventing both overcooling and overheating. The use of heaters may be twice as efficient, but cannot prevent overheating and indeed can cause overheating in low Earth orbit. Replacing the core with an insulating material prevents overheating and overcooling, but is less mass-efficient and is only effective in orbits with eclipses shorter than $1520 \mathrm{~s}$ (hot) $/ 1200 \mathrm{~s}$ (cold) in low Earth orbit and $1120 \mathrm{~s}$ (hot)/990 s (cold) in geosynchronous orbit. Louvers are potentially a mass-efficient solution, but have been shown to be feasible only in a cold low Earth orbit.

The upper limit on the length of eclipse that can be made a safe is determined by the amount of the MFPS savings that one is willing to sacrifice. In this respect, a heater is the preferred option at high altitudes, where overheating is not an issue. To clarify, using all of the MFPS savings as added phase-change material can enable orbits with eclipses up to $3500 \mathrm{~s}$, whereas this mass used as extra battery capacity for a heater enables orbits with eclipses up to $4000 \mathrm{~s}$.

\section{Conclusion}

Multifunctional power structures offer system-level mass benefits to spacecraft. An application of this technology is to embed a COTS battery into the structure of a deployed wing solar array. In this configuration, a key technical issue is the management of the temperature of the batteries. This issue defines the range of orbits in which MFPS can be used. Viability of a thermal-control system was defined by the feasibility of the TCS and what proportion of the MFPS savings were used by the added mass.

A numerical model of the solar-array MFPS was defined using a lumped-parameter method. This model was used to assess the viability of a range of thermal-control systems to extend the range of orbits in which MFPS can be used. Analysis of the unaltered structure revealed that overcooling during eclipse was the biggest threat. Extending the time it requires the batteries to overcool in eclipse extends the orbit range by enabling passage through longer eclipses. It was found that adjusting the optical properties of the coatings applied to the panel to reduce heat loss was the most efficient method as it requires very little added mass. The use of heaters, insulation, and PCMs is limited by their mass, though their use does provide a greater increase in the time to overcool, further increasing the range of viable orbits.

\section{Acknowledgments}

The authors wish to acknowledge the EPSRC for their contribution

\section{Notation}

The following symbols are used in this paper:

$A_{k}=$ cross-sectional area;

$A_{r}=$ area of surface;

$F_{\text {ij }}=$ view factor between surface and cold space;

$H=$ heat capacity;

$Q_{k}=$ conduction heat flow;

$Q_{r}=$ radiation heat flow;

$T=$ temperature;

$T_{i}, T_{j}=$ node temperatures;

$k=$ thermal conductivity;

$x=$ conduction path length;

$\varepsilon_{i}=$ emittance of the surface; and

$\varepsilon_{j}=$ emittance of cold space.

\section{References}

Anderson, B., Justus, C., and Batts, W. (2001). "Guidelines for the selection of near earth thermal environment parameters for spacecraft design." TM-2001-211221, National Aeronautics and Space Administration (NASA), Washington, DC.

Automation Creations. (2007). "Matweb." 〈http://www.matweb.com〉 (Jun. 26, 2007).

Chen, Y., and Evans, J. (2005). "Thermal analysis of lithium-ion batteries." J. Power Sources, 140(1), 111-124.

Choquette, Y., and Lessard-Deziel, D. (2002). "Proton and electron radiation effects on dry lithium metal polymer batteries." Proc., IEEE Radiation Effects Data Workshop, IEEE, New York, 120-126.

Foster, J., and Aglietti, G. (2010). "The thermal environment encountered in space by a multifunctional solar array." Aerosp. Sci. Technol., 14(3), 213-219.

Foster, J. A., Roberts, S. C., and Aglietti, G. S. (2008). "Multifunctional power structures and related thermal issues." Proc., SMASIS2008, American Society of Mechanical Engineers (ASME), New York.

Gilmore, D., ed., (2002). Spacecraft thermal control handbook, American Institute of Aeronautics and Astronautics (AIAA), Reston, VA.

Hawes, D., Feldman, D., and Banu, D. (1993). "Latent heat storage in building materials." Energy Build., 20(1), 77-86.

Hisano, K., Iwasaki, H., and Ishizuka, M. (1995). "Thermal analysis of notebook personal computer." Proc., IEEE/CPMT International Electronic Manufacturing Technology (IEMT) Symp., IEEE, New York, 456-459.

Ilic, D., Birke, P., Holl, K., Wohrle, T., Haug, P., and Birke-Salam, F. (2004). "PoLiFlex, the innovative lithium-polymer battery." J. Power Sources, 129(1), 34-37.

Justus, C., Batts, G. W., Anderson, B. J., and James, B. F. (2001). "Simple thermal environment model (STEM) user's guide." TM-2001-211222 NASA MSFC, National Aeronautics and Space Administration (NASA), Washington, DC.

Khateeb, S. A., Amiruddin, S., Farid, M., Selman, J. R., and Al-Hallaj, S. (2005). "Thermal management of Li-Ion battery with phase change material for electric scooters: Experimental validation." J. Power Sources, 142(1-2), 345-353.

Li, P., Cheng, H., and Qin, W. (2006). "Numerical simulation of temperature field in solar arrays of spacecraft in low earth orbit." Numer. Heat Transfer, Part A, 49(8), 803-820.

Megahed, A., and El-Dib, A. (2007). "Thermal design and analysis for battery module for a remote sensing satellite." J. Spacecr. Rockets, 44(4), 920-926.

Neudecker, B., Benson, M., and Emerson, B. (2003). "Power fibers: Thinfilm batteries on fiber substrates." Proc. 14th Int. Conf. Composite Materials (ICCM 14), Defense Advanced Research Projects Agency, Arlington, VA, 739-745.

Olson, J., Feaver, T., and Lyman, P. (2003). "Structural lithium-ion batteries using dual-functional carbon fabric composite anodes." Proc. 14th Int. Conf. Composite Materials (ICCM 14), Defense Advanced Research Projects Agency, Arlington, VA. 
Queheillalt, D. T., Carbajal, G., Peterson, G., and Wadley, H. N. (2008). "A multifunctional heat pipe sandwich panel structure." Int. J. Heat Mass Transfer, 51(1-2), 312-326.

Queheillalt, D. T., Katsumui, Y., and Wadley, H. (2001). "Electron beamDirected vapor deposition of multifunctional structures." Materials Research Society Symp.-Proc., Cambridge University Press, Cambridge, UK, 672, 05.6.1-O5.6.6.

Rawal, S. P. (1999). "Thermal management for multifunctional structures." IEEE Trans. Adv. Packag., 22(3), 379-383.

Reif, F. (1965). Fundamentals of statistical and thermal physics, McGrawHill Kogakusha, Tokyo.

Roberts, S., and Aglietti, G. (2006). "Battery performance degradation under vibration loading." 7th Int. Conf. Dynamics \& Control of Systems and Structures in Space, Cranfield Univ., Cranfield, UK.

Roberts, S., and Aglietti, G. (2007). "Multifunctional power structures for spacecraft application." Proc., 57th Int. Astronautical Congress, International Astronautical Federation, Paris.

Roberts, S. C., and Aglietti, G. S. (2008). "Satellite multifunctional power structure: Feasibility and mass savings." Proc. Inst. Mech. Eng., Part G, 222(1), 41-52.

Sharma, A., Tyagi, V., Chen, C., and Buddhi, D. (2009). "Review on thermal energy storage with phase change materials and applications." Renew. Sustain. Energ. Rev., 13(2), 318-345.

Snyder, J. F., Carter, R. H., and Wetzel, E. D. (2007). "Electrochemical and mechanical behavior in mechanically robust solid polymer electrolytes for use in multifunctional structural batteries." Chem. Mater., 19(15), 3793-3801.

United States Coast Guard (USCG). (1999). "Formic acid chemical hazards response information system datasheet." CAMEO Chemicals, National Oceanic and Atmospheric Administration, Washington, DC.

Wang, X., Kato, M., Naito, H., Yamada, C., Segami, G., and Kibe, K. (2006). "A feasibility study of commercial laminated lithium-ion polymer cells for space applications." J. Electrochem. Soc., 153(1), A89-A95.

Wiener, M., Reichenauer, G., Hemberger, F., and Ebert, H.-P. (2006). "Thermal conductivity of carbon aerogels as a function of pyrolysis temperature." Int. J. Thermophys., 27(6), 1826-1843. 